\title{
Differences in myoelectric manifestations of fatigue during isometric muscle actions
}

\author{
Piotr Gawda' ${ }^{1, A, C-D, F}$, Michał Ginszt ${ }^{1, A-B, D, F}$, Apolinary Ginszt ${ }^{1, D-F}$, Halina Pawlak ${ }^{2, E-F}$, Piotr Majcher ${ }^{1, A, E-F}$ \\ ${ }^{1}$ Medical Univesity, Lublin, Poland \\ ${ }^{2}$ University of Life Sciences, Lublin, Poland \\ A - Research concept and design, B - Collection and/or assembly of data, C - Data analysis and interpretation, \\ $D$ - Writing the article, E-Critical revision of the article, F-Final approval of article
}

Gawda P, Ginszt M, Ginszt A, Pawlak H, Majcher P. Differences in myoelectric manifestations of fatigue during isometric muscle actions. Ann Agric Environ Med. 2018; 25(2): 296-299. doi: 10.26444/aaem/81716

\begin{abstract}
I Abstract
Introduction. Muscle fatigue has been studied for a long time with the use of a wide variety of exercise models, protocols and assessment methods, among which surface electromyography (sEMG) is most commonly used. The main sEMG parameters (amplitude and frequency) are prevalently used to evaluate the level of muscle fatigue in static and dynamic contractions. Objective. The purpose of this study is to determine and compare 2 separate indices: IF1 basis of the sEMG signal amplitude analysis and IF2 basis of the SEMG median frequency analysis, related to muscles fatigue during an isometric contraction. Materials and method. The study was performed on 60 professional runners divided into 2 equal groups. The first group comprised sprinters, competing in short distance track and field events (100 and 200 meters). The second group consisted of middle-distance runners, competing in middle-distance track and field events (800 and 1,500 meters). The electrical activity of the VMO muscles of the right and left lower limbs was recorded simultaneously during isometric activity in a squatting position. The sEMG data was used to determine and compare $\mathrm{IF}_{1}$ and $\mathrm{IF}_{2}$ indices.

Results. During isometric measurement, sprinters presented a much more significant increase in the mean amplitude of $s E M G$ signal in comparison to middle-distance runners (mean $\mathrm{IF}_{1}$ difference: $0.228 ; p=0.007$ ). Analysis of the median frequency did not show significant differences between the 2 groups (mean $\mathrm{IF}_{2}$ difference: $0.037 ; \mathrm{p}=0.12$ ).

Conclusions. Change in sEMG amplitude during isometric exercise may be related to muscles fatigue. The use of fatigue indices, based on SEMG amplitude, as an objective indicator of the efficacy of an endurance training programme for sportsmen requires further research.
\end{abstract}

\section{Key words}

vastus medialis oblique, squatting, sEMG, fatigue

\section{INTRODUCTION}

Muscle fatigue, defined as the inability to maintain the required or expected force or power output, is a common symptom during sport activities [1]. Moreover, this symptom is present in many diseases and health conditions related to physical inactivity [2]. From the physiological point of view, fatigue is related to the exhaustion of the metabolic reserves and accumulation of by-products in the contracting muscle, which reduces its contractile properties [3]. Moreover, the physiological and anatomical factors, such as: muscle fibre composition (fast-twitch to slow-twitch muscle fibre ratio), neuromuscular characteristics, high-energy metabolite stores, buffering capacity, ionic regulation, capillarisation, and mitochondrial density, can determine the fatigue profile of a muscle [1]. Thus, the mechanism of fatigue is related to changes in both the muscle (peripheral fatigue) and the nerve system (central fatigue) [4].

Muscle fatigue has been studied for a long time with the use of a wide variety of exercise models, protocols and assessment methods. There are several methods (sonomyography, acoustic myography, surface mechanomyography), which have been used in studies on muscle fatigue [5, 6]. Moreover, kinematics and kinetics measurements, based on speed,

Address for correspondence: Michał Ginszt, Medical Univesity of Lublin, Poland Magnoliowa 2, 20-143 Lublin, Poland

E-mail: michal.ginszt@umlub.pl

Received: 21.06.2017; accepted: 28.12.2017; first published: 21.02.2018 velocity, acceleration of muscles contraction, joint angle and oscillations, are recorded as indicators of fatigue $[7,8]$. In addition, the commonly used tool in relation to muscle fatigue is surface electromyography (sEMG), which allows for non-invasive examination by means of surface electrodes [9]. The main sEMG parameters (amplitude and frequency) are prevalently used to evaluate the level of muscle fatigue in static and dynamic contractions [10]. One symptom of a muscle fatigue during an isometric contraction is the increase in the sEMG root mean square (RMS) amplitude signal, which is interpreted as the recruitment of additional motor units [11]. Another symptom of muscle fatigue during contraction is the shift in median frequency of a complete power range towards lower values [12]. Median frequency parameters are related to changes in muscle fibre conduction velocities and subsequent changes in the duration of the motor unit action potential waveform [13, 14, 15]. However, there are many factors that could influence the quality of the EMG signal (quantity of tissue between the electrode and the surface of the muscle, the depth and location of active fibres, the timing and intensity of muscle contraction, and the properties of the electrode and amplifier) [3]. Therefore, interpretation of EMG is not so straightforward and requires the correct selection of signal and indicator parameters to properly interpret the test results. 


\section{OBJECTIVE}

The purpose of this study is to determine and compare 2 separate indices ( $\mathrm{IF}_{1}$ basis of the sEMG signal amplitude analysis and $\mathrm{IF}_{2}$ basis of the sEMG median frequency analysis) related to muscles fatigue during an isometric contraction.

\section{MATERIALS AND METHOD}

Sixty professional runners, with a minimum experience of 3 years of training and practising at least 4 times per week in sports clubs, participated in the study. All athletes were competing at provincial level. Participants with a history of surgery and traumatic injuries to both lower extremity and spine in the previous 2 years were excluded. Runners were divided into 2 groups of even numeracy. The first group comprised sprinters, competing in short distance track and field events (100 and 200 meters; 23 males, 7 females; aged 23.9 \pm 5.1$)$. The second group consisted of middle-distance runners, competing in middle distance track and field events (800 and 1,500 meters; 23 males, 7 females; aged 24.1 \pm 4.5 ). Detailed characteristics of the participants are shown in Table 1. Both sprinters and middle-distance runners performed several types of training, including low/high-resistance training, plyometric training, and explosive training. Training loads were selected in order to meet established training goals (competing in short or middle distances races). During a 6-month period prior to the study, all participants subjects were in a good state of health, without any conditions which could influence the test results. All the tests were conducted in the morning to decrease the influence of the variability of bioelectrical activity of the muscle.

The study was approved by the Ethics Committee of the Medical University in Lublin, Poland (KE-0254/339/2014), and conducted in accordance with the Declaration of Helsinki. All participants gave written informed consent to participate.

Prior to data collection, each athlete performed a 15 minutes dynamic warm-up. After warm-up, participants were asked to assume a squatting position without shoes and with minimal clothing (feet hip width apart, knees bent at a $60^{\circ}$ angle, hands laced at the level of the chest), according to the procedure described by Hyong 2015 [16]. A goniometer was used to maintain the knee angle. During the test, each participant was asked to maintain the position for 60 seconds.

The electrical activity of the vastus medialis oblique (VMO) muscles of the right and left lower limbs was recorded simultaneously during isometric activity in a squatting position. The 4-channel Noraxon MyoTrace 400, compatible with Master Edition software, was used for the recording. The sampling rate was $1,000 \mathrm{~Hz}$, and frequency bandwidth $80-250 \mathrm{~Hz}$. Pairs of electrodes $(\mathrm{Ag} / \mathrm{AgCl})$ were attached at $4 / 5$ of the distance between the upper inner part of the knee bone and the anterior superior iliac spine on the VMO muscles in relation to the muscle fibre direction [17]. In compliance with SENIAM standards, the reference electrode was placed over the patella bone [18]. The skin at the attachment sites was shaved and cleansed with $90 \%$ ethyl alcohol solution.

In order to determine the $\mathrm{IF}_{1}$ value, the raw sEMG data were full wave rectified. Root mean square values were calculated using a $100 \mathrm{~ms}$ sliding window. The $\mathrm{IF}_{1}$ was determined by division of the mean amplitude of the last 3 seconds (57th
- 60th seconds) through the mean amplitude of the first 3 seconds of contraction ( 0 - 3rd seconds). The raw sEMG signal was used for analysis of the median frequency. The $\mathrm{IF}_{2}$ was determined by division of the median frequency of the first and last seconds of the test. The sEMG values from dominant leg were used to statistical analysis. The dominant leg was determined by asking the subjects which leg they would choose to kick a ball, according to the Van Lunen et al. 2003 protocol [19].

IBM SPSS STATISTICS 21 programme was used to prepare the statistical analysis. The data was tested for normal distribution, by a Shapiro-Wilk test of normality, which showed normal distributed material. Thus, to evaluate the significance of the analysed variables, T-test independent samples were used. Differences were regarded as statistically significant if the level of probability value was lower than the statistical significance $(\mathrm{p}<0.05)$.

\section{RESULTS}

The characteristics of groups analysis did not show significant difference ( $p>0.05$ ) in age, height and body weight between sprinters and middle - distance runners (Tab. 1).

Table 1. Demographic data for study participants

\begin{tabular}{lcccccc}
\hline Group & $\mathrm{n}$ & Male & Female & $\begin{array}{c}\text { Age } \\
(\text { years })^{*}\end{array}$ & $\begin{array}{c}\text { Height } \\
(\mathrm{cm})^{*}\end{array}$ & $\begin{array}{c}\text { Body weight } \\
(\mathrm{kg})^{*}\end{array}$ \\
\hline Sprinters & 30 & 23 & 7 & $23.9 \pm 5.1$ & $173.8 \pm 5.5$ & $67.3 \pm 4.8$ \\
\hline $\begin{array}{l}\text { Middle-distance } \\
\text { runners }\end{array}$ & 30 & 23 & 7 & $24.1 \pm 4.5$ & $174.6 \pm 5.8$ & $65.5 \pm 6.1$ \\
\hline
\end{tabular}

* $p<0.05$ (independent samples $T$ - test, $p=0.05$ )

During squatting test, sprinters presented a much more significant increase in the mean amplitude of sEMG signal $\left(\mathrm{IF}_{1}: 1.391 \pm 0.079\right)$, compared to middle-distance runners $\left(\mathrm{IF}_{1}: 1.163 \pm 0.085\right)$. Analysis showed significant difference $(\mathrm{p}<0.05)$ in $\mathrm{IF}_{1}$ between the 2 groups (Tab. 2$)$.

Table 2. Difference in the SEMG mean amplitude $\left(I_{1}\right)$ of $V M O$ muscles between sprinters and middle-distance runners during squad test

Runners $\quad \mathrm{n} \quad$ Mean IF $_{1}$ SD $\quad$ Mean Difference $\quad t \quad r$

$\begin{array}{llll}\text { Sprinters } & 30 & 1.391 & 0.079\end{array}$

$0.228 \quad 10.787 \quad 0.007^{*}$

$\begin{array}{llll}\begin{array}{l}\text { Middle- distance } \\ \text { runners }\end{array} & 30 & 1.163 & 0.085\end{array}$

* statistically significant results (independent samples $\mathrm{T}$ - test, $\mathrm{p}=0.05$ )

In sprinters, analysis did not show significant difference in the median frequency of the sEMG signal $\left(\mathrm{IF}_{2}\right)$ of VMO muscles, compared to middle-distance runners (Tab. 3). 
Table 3. Difference in the sEMG median frequency (IF2) of VMO muscles between sprinters and middle-distance runners during squad test

\begin{tabular}{|c|c|c|c|c|c|c|}
\hline Runners & $\mathrm{n}$ & Mean $\mathrm{IF}_{2}$ & SD & Mean Difference & $\mathrm{t}$ & $\mathrm{p}$ \\
\hline Sprinters & 30 & 1.251 & 0.096 & \multirow{2}{*}{0.037} & \multirow{2}{*}{1.577} & \multirow{2}{*}{0.12} \\
\hline Middle- distance runners & 30 & 1.213 & 0.088 & & & \\
\hline
\end{tabular}

\section{DISCUSSION}

An objective examination and determination of muscle fatigue level is extremely important in terms of monitoring the effects of athlete training. Applying objective methods of measuring muscle fatigue provises information on whether an athlete is adapting to a training programme. In addition, the correct interpretation of fatigue indices helps to reduce the risk of sportsman overload [20]. Determination of fatigue indices is possible through the use of surface electromyography, but the interpretation of the results of sEMG is still a matter of reflection [21].

Both the mean amplitude and the median frequency of the sEMG signal were measured to determine the effect of muscles fatigue $[4,11,14,22]$. The sEMG amplitude increases during submaximal isometric contractions, in comparison to median frequency, which decrease during isometric fatiguing contraction [15].

It was recently found that the changes due to fatigue in the sEMG signal (increased amplitude and decreased frequency), suggesting that the recruitment of motor units (MU) firing rates correlates with sEMG amplitude and frequency [11]. The central nervous system regulates muscle force production during fatigue conditions by varying 2 main motor unit parameters: the recruitment of new motor units, and the modulation of firing rates of active motor units [23]. Studies have shown that MU firing rate decreases with muscles fatigue, which can also be observed in the amplitude and frequency of sEMG $[24,25]$. However, MU recruitment cannot be used synonymously with sEMG amplitude and cannot be inferred from changes in sEMG amplitude [21].

In the presented study, in order to compare 2 fatigue indices, 2 different groups of runners were selected which differed significantly in the type of training. A considerable increase in the mean amplitude of sEMG signal was observed for sprinters, compared to middle-distance runners. The lower increase of sEMG amplitude in middle-distance athletes can be interpreted as a greater resistance to muscle fatigue during isometric contraction. In this study, analysis did not show significant difference in spectral median frequency between the 2 groups of runners. Thus, the obtained results suggest that sEMG amplitude better reflects differences in muscle fatigue between sprinters and middle-distance runners. However, the mechanism of increase in amplitude during isometric contraction cannot be based solely on MU recruitment and needs further investigation [26]. Moreover, the athletic activity of runners takes place in dynamic conditions and cannot be unequivocally assessed during isometric tests. The use of dynamic contractions in sEMG measurement is more relevant to muscles function during running; however, the shifts of the underlying muscle fibres with respect to the recording surface electrodes affect sEMG measurement during dynamic contractions [27, 28]. Hence, in the presented study, isometric activity was used which is more reliable and easier to analyze.

\section{CONCLUSIONS}

1. Change in sEMG amplitude during isometric exercise may be related to muscles fatigue.

2. The use of fatigue indices, based on sEMG amplitude, as an objective indicator of the efficacy of an endurance training programme for sportsmen requires further research.

\section{REFERENCES}

1. Bogdanis GC. Effects of Physical Activity and Inactivity on Muscle Fatigue. Front Physiol. 2012; 3: 142.

2. Rimmer JH, Schiller W, Chen M-D. Effects of disability-associated low energy expenditure deconditioning syndrome. Exerc Sport Sci Rev. 2012; 40(1): 22-9.

3. Al-Mulla MR, Sepulveda F, Colley M. A Review of Non-Invasive Techniques to Detect and Predict Localised Muscle Fatigue. Sensors. 2011; 11(4): 3545-94.

4. Zając A, Chalimoniuk M, Maszczyk A, Gołaś A, Lngfort J. Central and Peripheral Fatigue During Resistance Exercise - A Critical Review. J Hum Kinet. 2015; 49: 159-69.

5. Shi J, Chang Q, Zheng Y-P. Feasibility of controlling prosthetic hand using sonomyography signal in real time: preliminary study. J Rehabil Res Dev. 2010; 47(2): 87-98.

6. Orizio C, Gobbo M, Diemont B, Esposito F, Veicsteinas A. The surface mechanomyogram as a tool to describe the influence of fatigue on biceps brachii motor unit activation strategy. Historical basis and novel evidence. Eur J Appl Physiol. 2003; 90(3-4): 326-36.

7. Guo J-Y, Zheng Y-P, Huang Q-H, Chen X. Dynamic monitoring of forearm muscles using one-dimensional sonomyography system. J Rehabil Res Dev. 2008; 45(1): 187-95.

8. Vedsted P, Blangsted AK, Søgaard K, Orizio C, Sjøgaard G. Muscle tissue oxygenation, pressure, electrical, and mechanical responses during dynamic and static voluntary contractions. Eur J Appl Physiol. 2006; 96(2): 165-77.

9. Subbu R, Weiler R, Whyte G. The practical use of surface electromyography during running: does the evidence support the hype? A narrative review. BMJ Open Sport Exerc. Med. 2015; 1(1): e000026.

10. Donaldson S, Donaldson M, Snelling L. SEMG evaluations: an overview. Appl Psychophysiol Biofeedback. 2003; 28(2): 121-7.

11. Calder KM, Stashuk DW, McLean L. Physiological characteristics of motor units in the brachioradialis muscle across fatiguing lowlevel isometric contractions. J Electromyogr Kinesiol Off J Int Soc Electrophysiol Kinesiol. 2008; 18(1): 2-15.

12. Farina D, Merletti R. Comparison of algorithms for estimation of EMG variables during voluntary isometric contractions. J. Electromyogr. Kinesiol. Off. J. Int. Soc. Electrophysiol. Kinesiol. 2000; 10(5): 337-49.

13. Allison GT, Fujiwara T. The relationship between EMG median frequency and low frequency band amplitude changes at different levels of muscle capacity. Clin Biomech Bristol Avon. 2002; 17(6): 464-9.

14. Abboud J, Nougarou F, Descarreaux M. Muscle activity adaptations to spinal tissue creep in the presence of muscle fatigue. PLoS ONE. 2016; 11:e0149076. 10.1371/journal.pone.0149076.

15. González-Izal M, Malanda A, Gorostiaga E, Izquierdo M. Electromyographic models to assess muscle fatigue. J Electromyogr Kinesiol Off J Int Soc Electrophysiol Kinesiol. 2012; 22(4): 501-12.

16. Hyong IH. Effects of squats accompanied by hip joint adduction on the selective activity of the vastus medialis oblique. J Phys Ther Sci. 2015; 27(6): 1979-81.

17. Park J, Lee D, Kim J-S, Hong J-H, You J-H, Park I. Effects of visibility and types of the ground surface on the muscle activities of the vastus medialis oblique and vastus lateralis. J Phys Ther Sci. 2015;27(8): 2435-7.

18. Hermens HJ, Freriks B, Disselhorst-Klug C, Rau G. Development of recommendations for SEMG sensors and sensor placement procedures. J Electromyogr Kinesiol. 2000; 10(5): 361-74.

19. Van Lunen BL, Roberts J, Branch JD, Dowling EA. Association of Menstrual-Cycle Hormone Changes with Anterior Cruciate Ligament Laxity Measurements. J Athl Train. 2003; 38(4): 298-303.

20. Halson SL. Monitoring Training Load to Understand Fatigue in Athletes. Sports Med Auckl Nz. 2014; 44(Suppl 2): 139-47.

21. Vigotsky AD, Beardsley C, Contreras B, Steele J, Ogborn D, Phillips SM. Greater electromyographic responses do not imply greater motor unit recruitment and 'hypertrophic potential' cannot be inferred. J Strength Cond Res. 2017; 31(1): 1-4. doi: 10.1519/JSC.0000000000001249. 
22. Stania M, Chmielewska D, Kwaśna K, Smykla A, Taradaj J, Juras G. Bioelectrical activity of the pelvic floor muscles during synchronous whole-body vibration - a randomized controlled study. BMC Urol. 2015; 15: 107.

23. Contessa P, Adam A, De Luca CJ. Motor unit control and force fluctuation during fatigue. J Appl Physiol. 2009; 107(1): 235-43.

24. Mottram CJ, Jakobi JM, Semmler JG, Enoka RM. Motor-unit activity differs with load type during a fatiguing contraction. J Neurophysiol. 2005; 93(3): 1381-92.

25. Balshaw TG, Pahar M, Chesham R, Macgregor LJ, Hunter AM. Reduced firing rates of high threshold motor units in response to eccentric overload. Physiol Rep. 2017; 5(2): e13111. doi: 10.14814/phy2.13111.
26. Enoka, RM, Duchateau J. Inappropriate interpretation of surface EMG signals and muscle fiber characteristics impedes understanding of the control of neuromuscular function. J Appl Physiol. 2015; 119(12): 1516-8.

27. Farina D. Interpretation of the surface electromyogram in dynamic contractions. Exerc Sport Sci Rev. 2006; 34(3): 121-7.

28. Farina D, Merletti R, Nazzaro M, Caruso I. Effect of joint angle on EMG variables in leg and thigh muscles. IEEE Eng. Med. Biol. Mag. 2001; 20(6): 62-71. 